

\title{
Hipótesis de convergencia absoluta en el caso de las entidades federativas de México, 2003-2016
}

Absolute convergence hypothesis in the case of the federative entities of Mexico, 2003-2016

\author{
Ricardo Alan Solorzano Mendoza \\ Universidad Autónoma Metropolitana (México) \\ Autor para correspondencia: alan_1193@hotmail.com \\ Eddy Lizarazu Alanez \\ Universidad Autónoma Metropolitana, Unidad Iztapalapa (México)
}

(Recibido: 17/02/2020. Aceptado para publicación: 03/09/2020)

DOI: $10.22201 / f e .24484962 e .2020 .9 .15 .4$

\section{RESUMEN}

El presente trabajo tiene por objetivo analizar la hipótesis de convergencia absoluta del ingreso de forma empírica y teórica, para ello se presenta como referente una recapitulación sobre la formalización del modelo de Solow, con él se pretende que se identifiquen las interrelaciones determinantes del estado estacionario, para posteriormente hacer una revisión teórica del fenómeno de convergencia con el objetivo de realizar una investigación empírica utilizando los datos del ingreso per cápita de las entidades federativas de México, lo anterior bajo el análisis descriptivo de la crisis económica de 2008. El artículo se desenvuelve sobre los tres aspectos anteriormente mencionados: la teoría económica sobre la predicción del fenómeno de convergencia, la teoría sobre el fenómeno en sí mismo y la evidencia empírica usando a la economía de México para el análisis.

Palabras clave: convergencia absoluta, crecimiento económico, modelo de Solow.

\section{ABSTRACT}

The present investigation has the objective to analyze the hypothesis of absolute convergence of the per capita income in an empirical and theoretical form, for it is presented a recapitulation about the formalization of the Solow model, whit this is pretended to identify the interrelationships that determine the steady state, and whit this theoretical base is going to be realized an empirical investigation using the data of the per capita income of the entities of México in the context of the economic crisis of 2008. The methodology of the analysis consist in present the three aspects before mentioned: The economic theory on the prediction of the phenomenon of convergence, the theory about the phenomenon itself, and the empirical evidence using the economy of Mexico for the analysis.

Keywords: Absolute convergence, economic growth, Solow model.

JEL Classification: C13, O41, O47.

Clasificación JEL: C13, O41, O47. 


\section{INTRODUCCIÓN}

$\mathbf{L}$

a hipótesis de convergencia del ingreso es un corolario del modelo de Solow (1956), ya que las interrelaciones determinantes del estado estacionario implican teóricamente el fenómeno de convergencia, en particular a lo referente a la tasa de crecimiento. En este aspecto, el trabajo que es una referencia para la investigación empírica estatal de México es Esquivel (1999), que llega a la conclusión de que efectivamente hay una disminución en la dispersión del ingreso para el periodo de 1940-1995. Sin embargo, esto no es un tema agotado, pues en la medida de mayor disponibilidad de datos se van generando nuevos análisis, por ejemplo, aquellos que no necesariamente tienen el interés analítico sobre la dinámica entre-estatal, y que pueden centrar su análisis al interior de un estado sin abandonar la base teórica del modelo de Solow, ejemplo de esto es Vergara, Martínez y Mejía (2010) que hacen un análisis regional del Estado de México.

Los aspectos más relevantes de la reflexión de este artículo son la teoría económica sobre el fenómeno de convergencia absoluta y la evidencia empírica para el caso de México. El problema de investigación se centra en contrastar si las conclusiones sobre la tasa de crecimiento a las que arriba el modelo de Solow son coherentes con la evidencia empírica emanada de las entidades federativas, por lo cual, la discusión analítica se basa en tres aspectos fundamentales: la teoría del crecimiento económico de Solow, la hipótesis de convergencia y, por último, el análisis empírico.

En términos de la teoría económica se hace una exposición de la formalización del modelo de Solow bajo los enfoques más utilizados en su enseñanza, que por lo general incluyen una función de producción tipo Cobb-Douglas, la tasa de depreciación del capital, de ahorro y el crecimiento poblacional; asimismo, en este análisis se indica la consecuencia de introducir (o no) al progreso tecnológico como una variable explicativa, lo anterior se hace menester para entender que la hipótesis de convergencia absoluta es una implicación de la existencia de un estado estacionario, esta idea es el nexo que conecta la teoría del crecimiento con las especificaciones econométricas denominadas convergencia- $\beta$ absoluta y convergencia- $\alpha$, mismas con las que se puede identificar empíricamente la existencia de convergencia. Por último, en términos del análisis práctico, comprobamos si la base teórica del modelo de Solow es adecuada como enfoque para describir el proceso de convergencia regional.

\section{LA TEORÍA DEL CRECIMIENTO ECONÓMICO DE SOLOW}

Para entender el concepto de convergencia absoluta desde el enfoque de la teoría ne clásica necesariamente tiene que recurrirse a las modelizaciones subsecuentes al plaNteamiento original de Solow (1956), quien inicialmente publicó su modelo como una contestación teórica al modelo keynesiano de Harrod-Domar. Estas formalizaciones posteriores son esenciales, pues introdujeron la depreciación del capital en la ecuación de la evolución del producto y una función específica de producción. La especificación del modelo (de manera general ${ }^{1}$ ) se muestra en el cuadro 1.

Sin considerar el análisis en unidades efectivas de trabajo como lo hace Romer (2002). 


\section{CUADRO 1 \\ CONFORMACIÓN GENERAL DE LA MODELIZACIÓN SUBSECUENTE DEL MODELO DE SOLOW}

\begin{tabular}{|c|c|c|c|}
\hline Ecuación & Términos agregados & Términos per cápita* & Descripción \\
\hline $\begin{array}{l}\text { [01] Función de } \\
\text { producción }\end{array}$ & $Y_{t}=K_{t}^{\alpha} L_{t}^{1-\alpha}$ & $y_{t}=k_{t}^{\alpha}$ & $\begin{array}{l}\text { En términos agregados se } \\
\text { cumple homogeneidad de } \\
\text { grado uno y existen dos } \\
\text { factores: capital (físico) y } \\
\text { trabajo; cumple las condi- } \\
\text { ciones Inada }\end{array}$ \\
\hline $\begin{array}{l}\text { [02] lgualdad del } \\
\text { producto interno bruto } \\
\text { y el gasto agregado }\end{array}$ & $Y_{t}=C_{t}+I_{t}$ & $y_{t}=c_{t}+i_{t}$ & $\begin{array}{l}\text { No considera el gasto de } \\
\text { gobierno ni las exportacio- } \\
\text { nes netas** }\end{array}$ \\
\hline [03] Consumo & $C_{t}=(1-s) Y_{t}$ & $c_{t}=(1-s) y_{t}$ & $\begin{array}{l}\text { Los agentes siempre } \\
\text { consumen una fracción } \\
\text { constante de su renta }\end{array}$ \\
\hline [04] Inversión neta & $\dot{K}_{t}=I_{t}-\delta K_{t}$ & $\dot{k}_{t}=i_{t}-(n+\delta) k_{t}$ & $\begin{array}{l}\text { Es la resta de la inversión } \\
\text { bruta y la depreciación del } \\
\text { capital a una tasa cons- } \\
\text { tante } \delta\end{array}$ \\
\hline $\begin{array}{l}\text { [05] Tasa de crecimiento } \\
\text { del factor trabajo }\end{array}$ & $L_{t}=L_{0} e^{n t}$ & $* * *$ & La tasa es una constante \\
\hline \multicolumn{4}{|c|}{ Variables } \\
\hline Plazo & Clasificación & Términos agregados & Términos per cápita* \\
\hline \multirow{3}{*}{ Corto } & Exógenas: & $K_{t}, L_{t}$ & $k_{t}$ \\
\hline & & & \\
\hline & Endógenas: & $Y_{t}, C_{t}, I_{t}, \dot{K}_{t}, \dot{L}_{t}$ & $y_{t}, c_{t}, i_{t}, \dot{k}_{t} \neq 0$ \\
\hline \multirow{3}{*}{ Largo } & Exógenas: & $\dot{K}_{t}, \dot{L}_{t}$ & $\dot{k}_{t}=0$ \\
\hline & & & \\
\hline & Endógenas: & $Y_{t}, C_{t}, I_{t}, K_{t}, L_{t}$ & $y_{t}, c_{t}, i_{t}, k_{t}$ \\
\hline Parámetros: & & $s, \alpha, n, \delta$ & \\
\hline
\end{tabular}

Notas:

* Sala-I-Martin hace el supuesto de que la población es idéntica a la cantidad de trabajadores.

** No es una limitante al modelo, ya que se busca analizar en el largo plazo el papel de la inversión, y en específico el papel de la acumulación de capital.

** En el análisis per cápita puede considerarse el crecimiento de la población como un parámetro dado, es decir, no necesariamente debe representarse una ecuación estructural, esto es válido siempre que todas las variables ya estén representadas en términos per cápita, pero aun así puede describirse que el parámetro proviene de considerar $\dot{L}_{t} / L_{t}=n$. Fuente: elaboración propia a partir de lo expuesto por Sala-I-Martin (2000). 
Analizando en términos per cápita y sustituyendo [03] en [02] se obtiene que la inversión es igual a la tasa de ahorro por el nivel de producto, si se sustituye a [01] en esa igualdad se tendrá que $i_{t}=s k_{t}^{\alpha}$, misma expresión que al sustituir en [04] dará como resultado lo que se conoce como la ecuación fundamental del modelo de Solow, que en realidad es la inversión neta en términos del capital, que al dividirse entre este último, generará la ecuación de la tasa de crecimiento $\left(\dot{k}_{t} / k_{t}\right)=s k_{t}^{\alpha-1}-(n+\delta)$. Dados los rendimientos decrecientes del capital, conforme menor sea el nivel de este factor la tasa de crecimiento del mismo tenderá a ser infinita, en caso contrario, la tasa tenderá a ser ínfima (o incluso negativa). Asimismo, cuando se esté en el estado estacionario se cumple que $\left(\dot{k}_{t} / k_{t}\right)=0$.

\section{INTRODUCCIÓN DE LA TECNOLOGÍA AL MODELO DE SOLOW}

La tecnología puede incluirse en la función de producción, generalmente asumiéndose que al igual que el factor trabajo tiene una tasa de crecimiento exponencial, es decir, $A_{t}=A_{0} e^{g t}$ o bien puede ser simplemente incluida como una constante. Añadiéndose como una variable exógena en la ecuación [01] puede generarse un análisis en unidades efectivas de trabajo como lo hace Romer (2002), sin modificar en absoluto la esencia explicativa de las ecuaciones anteriormente especificadas, sólo considerando que la ecuación fundamental del modelo de Solow estaría conformada por $\dot{k}_{t}=s y_{t}-(n+\delta+g) k_{t}$.

Si la tecnología no es considerada, o solamente lo es como una constante, se puede comprobar que la tasa de crecimiento del producto per cápita en el estado estacionario sería nula, ya que de hecho esta tasa es proporcional a la tasa de crecimiento del capital. En un análisis de unidades efectivas de trabajo y considerando a la tecnología como una variable exógena (con tasa de crecimiento exponencial) se obtiene que la tasa de crecimiento del capital y del producto en el estado estacionario sería la misma, ${ }^{2}$ esto es, se obtiene que las tasas de crecimiento de ambos términos sería igual a $g$. En resumen, se incluya o no a la tecnología como una variable, se tendrá que la economía tenderá al estado estacionario $k^{\star}$, estipulado en el punto en que se dé la igualdad entre los términos que componen la ecuación de la tasa del crecimiento del capital.

\section{LA HIPÓTESIS DE CONVERGENCIA}

Barro, Grilli y Febrero (1997, p. 336), al igual que Romer (2002) y Sala-I-Martin (2000), utilizan los términos de economías pobres $y$ ricas para explicar la hipótesis de convergencia en el modelo de Solow, indicando que el proceso de convergencia radica en que las primeras "alcanzarían" a las segundas siempre que exista un estado estacionario común. Pero esta explicación es inexacta, porque en realidad puede que una economía pobre llegue antes al estado estacionario, y en ese caso debería indicarse que es la economía más rica la que alcanzase a una que inicialmente tuviera un menor nivel de capital y producto per cápita (denominada como economía pobre), aunque en ese caso debería comprobarse una tasa de crecimiento negativa por parte del país rico. Respecto a la hipótesis de convergencia, Barro, Grilli y Febrero (1997) concluyen que en el modelo de Solow este fenómeno es una "predicción

2 Estas pueden calcularse a partir de la definición de dichas variables en términos de unidades efectivas de trabajo. 
fundamental". Por su parte, Sala-I-Martin (2000) hace referencia a que las economías verificarán convergencia absoluta si estas tienen un mismo estado estacionario, esto se aprecia en el siguiente extracto: "el modelo neoclásico que acabamos de esbozar sólo predice la existencia de una relación negativa entre la renta y las tasas de crecimiento, en el caso de que la única diferencia entre los países resida en sus stocks iníciales de capital" (Sala-I-Martin, 2000, p. 46), es decir, que si existen diferencias estructurales significativas, entonces se tendrá que el modelo no predice un mayor crecimiento para los países menos ricos. Sin embargo, el modelo predice convergencia condicional "en el sentido de que la tasa de crecimiento de una economía está directamente relacionada con la distancia a la que se sitúa de su estado estacionario" (Sala-I-Martin, 2000, p. 47).

En realidad, el fenómeno de convergencia bajo el enfoque del modelo de Solow consiste en que economías con un mismo estado estacionario (por consiguiente con una igual tasa de ahorro, de crecimiento de la población y de depreciación), pero con distintos valores iníciales de capital y, por tanto, de ingreso per cápita, igualarían sus respectivos niveles de estos últimos cuando lleguen al estado estacionario; lo anterior como consecuencia de la propia dinámica de la evolución del capital, esta última revelada por los componentes de su tasa de crecimiento (la inversión y la inversión de reposición).

En cuanto a la investigación teórica, se ha establecido un cierto consenso de llamar convergencia absoluta (en contraposición a la hipótesis de convergencia condicional) cuando se verifica la igualación del ingreso sin necesidad de tomar en cuenta el grado de similitud que exista entre las economías estudiadas. Para el análisis empírico, la convergencia absoluta hace referencia a que existe un único intercepto en una regresión cuya variable dependiente sea la tasa de crecimiento y la independiente sea su rezago o el nivel de ingreso en su periodo inicial, ${ }^{3}$ y que cumpla que el parámetro asociado a esta regresión sea negativo.

Como lo hacen notar Durlauf y Johnson (2018, p. 2247), la convergencia condicional es aquella que se verifica cuando se toman en cuenta las características estructurales de las economías, ya que estas pudieran tener una diferencia estadísticamente significativa en alguno de los determinantes de la tasa de crecimiento en su dinámica de transición al largo plazo, pero que al tomar en cuenta esas características estructurales pudiera encontrarse evidencia estocástica para determinar la existencia de convergencia.

Lo anterior puede generalizarse siguiendo a Romer (2002), pues explica que para la comprobación de convergencia sólo es necesario que los países con niveles superiores de renta en su nivel inicial expongan un menor crecimiento económico durante el periodo de estudio. Aunque como se expondrá más adelante, esto debe confirmarse con un análisis de la dispersión del ingreso.

\section{ESPECIFICACIONES EMPÍRICAS}

\subsection{Convergencia $\beta$ absoluta}

Para identificar la existencia del fenómeno de convergencia econométricamente se busca mostrar una relación significativa de que "economías con un menor nivel de ingreso crecerán más rápido que aquellas con un nivel de ingreso más alto" (Durlauf y Johnson,

3 Dependiendo si se parte de un análisis de corte transversal o de datos panel. 
2018, p. 2247). Si la hipótesis de convergencia resulta ser cierta, entonces se habrá hallado evidencia de que en el largo plazo las brechas en el ingreso se van a eliminar. Lo anterior teóricamente será en virtud de que sólo existe un nivel de ingreso que es compatible con el estado estacionario en común.

En términos de variables discretas se puede plantear una regresión para investigación empírica, ésta se presenta de la siguiente manera (veáse el cuadro 2):

$$
\log \left(\frac{y_{i, t}}{y_{i, 0}}\right)=\alpha+\beta\left(\log \left(y_{i, 0}\right)\right)+u_{i}
$$

Si se encuentra significativo el parámetro $\beta$ y este es negativo, entonces ello quiere decir que existe convergencia absoluta, ya que esto indica que hay una relación inversa entre el crecimiento per cápita del ingreso y su nivel inicial. Por la especificación, el parámetro $\beta=\partial \log \left(y_{i, t} / y_{i, 0}\right) / \partial \log \left(y_{i, 0}\right)$ se interpreta en puntos porcentuales y se define como "la tasa instantánea de convergencia al estado estacionario" (Esquivel, 1999, p. 742).

El término de error estocástico juega un papel muy preponderante, ya que de manera previa al análisis del coeficiente de conver- gencia debe verificarse la distribución normal de este término, esto es así para que se pueda sustentar la significancia estadística del parámetro $\beta$, ya que si no se cumple este supuesto de normalidad, entonces la prueba convencional del estadístico $t$ no será válida.

\subsection{Convergencia $\sigma$}

Quah (1993) demostró que la existencia de una relación inversa entre la tasa de crecimiento y su nivel inicial era "una condición necesaria pero no suficiente para generar reducción en la dispersión del ingreso per cápita, que es, en último término, a lo que se refiere la hipótesis de la convergencia absoluta (...). Para incorporar la crítica de Quah en el análisis Barro y Sala-I-Martin acuñaron $(. .$.$) [el término] convergencia \sigma$ para referirse a la reducción en la dispersión del ingreso per cápita" (Esquivel, 1999, p. 728).

En general "entre un grupo de entes territoriales existe una tendencia a la convergencia tipo $\sigma$ si la dispersión del PIB [producto interno bruto] per cápita entre los mismos tiende a reducirse en el tiempo. Esta dispersión suele medirse mediante la desviación estándar" (Claure y Martínez, 2016, p. 29), por lo que para hacer un análisis econométrico

CUADRO 2

VARIABLESY PARÁMETROS DE LA REGRESIÓN

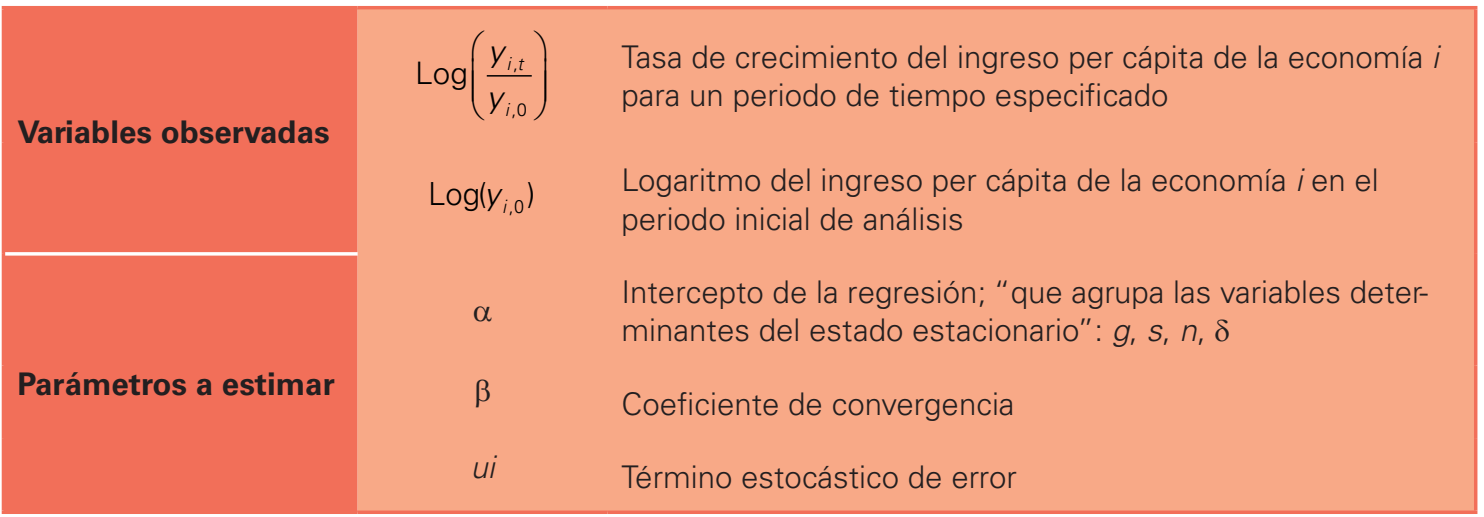

Fuente: elaboración propia a partir de Durlauf y Johnson (2018). 
de la existencia de este tipo de convergencia se debe estimar la siguiente regresión (véase el cuadro 3):

$$
\sigma_{t}=\alpha+\gamma t+\varepsilon_{t}
$$

Para verificar convergencia $\sigma$ es necesario comprobar la presencia de una reducción progresiva del ingreso conforme avanzan los periodos, esto es, que matemáticamente debe cumplirse que $\sigma_{t}<\sigma_{t-1}$. En este caso será necesario que el término de tendencia determinística de la regresión sea negativo, $\gamma$ $<0$,y también debe confirmarse que este sea un parámetro estadísticamente significativo.

\section{TRABAJOS EMPÍRICOS DE CONVERGENCIA ESTATAL}

En la actualidad existen una gran variedad de trabajos sobre convergencia, en muchos de ellos el análisis de esta hipótesis se hace considerando datos de distintos países, de hecho, como se aprecia en los textos de Mankiw (2000), Romer (2002), Barro y Martin-I-Sala (2004) y otros autores importantes presentan sus explicaciones utilizando los términos de "países", sin embargo, como es fácil de deducir, el análisis de convergencia no está solamente limitado a un carácter estrictamente internacional.

Por su importancia, cabe mencionar el texto de Barro et al. (1991) que toma en consideración el análisis no entre países sino directamente entre estados de diferentes regiones de Estados Unidos. Es a partir de las estimaciones realizadas que estos autores concluyen que para el caso de este país no se encontraron variaciones importantes del estado estacionario entre las economías, incluso hacen referencia a que estas variaciones pudieran ser más significativas en el caso de países con características similares, como lo son "una muestra de 98 países de la OCDE [Organización para la Cooperación y el Desarrollo Económicos]" (Barro et al., 1991, p. 112), que paradójicamente en otros textos, al tomar a países estructuralmente análogos, es cuando más evidencia se ha encontrado de convergencia absoluta, misma que implicaría que las economías examinadas no deberían mostrar diferencias en el estado estacionario.

Un trabajo pionero de investigación empírica estatal para el caso de México es el de Esquivel (1999); no obstante, por la falta de datos oficiales, en el conjunto de las observaciones se realizaron suposiciones con trascendentes repercusiones para las conclusio-

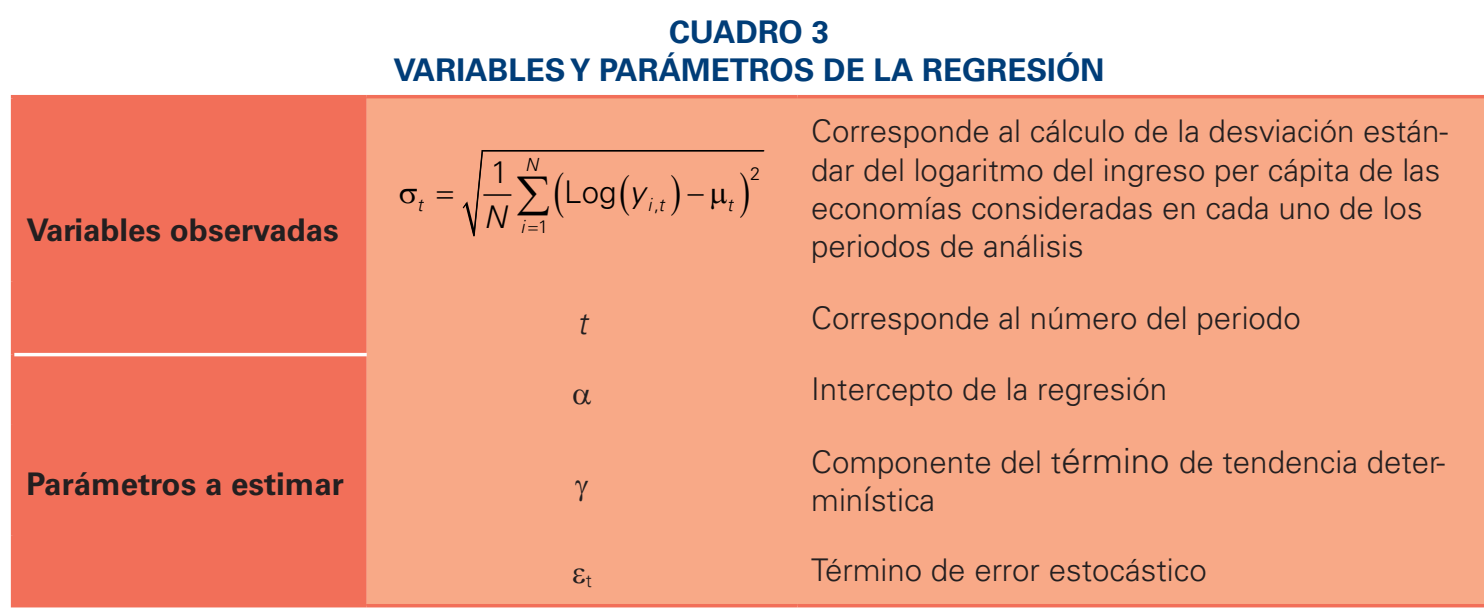

Fuente: elaboración propia con información de Garza (2006). 
nes, como la de mantener el supuesto de que "la producción estatal permaneció constante entre 1988 y 1990" (Esquivel, 1999, p. 11). De este mismo problema adolecen varios trabajos de convergencia sobre México, como es el caso de Camberos y Bracamontes (2016), quienes exponen un periodo de análisis muy amplio (de 1940 a 2013) pero sus datos están basados en estimaciones emanadas del uso de la técnica Cochrane Orcutt del trabajo de German (2005), lo que intrínsecamente habrá generado pérdida de información.

Entre los trabajos más actuales destacan aquellos que utilizan una metodología de datos panel, particularmente los referidos a las regresiones de mínimos cuadrados generalizados con variables instrumentales, lo anterior habitualmente en un marco dinámico como los especificados por Arellano y Bond (1991), Arellano y Bover (1995), Blundell y Bond (2000); sin embargo, estos modelos pueden tener sesgo, como lo demostró de forma general Cermeño (1998) o como también se muestra de manera particular en un análisis de convergencia estatal de México en Cermeño (2001). Estos modelos tienen además el inconveniente de considerar la existencia de los llamados efectos inobservados, de los cuales sólo se puede especular su existencia o, en su caso, su condición estocástica o determinística, que a su vez, de acuerdo con la concepción econométrica de Durlauf y Johnson (2018), implicaría un análisis de convergencia condicional.

Cabe mencionar que se pueden hallar más tipos de estudios empíricos sobre la convergencia, ejemplos de ello son los análisis de distribuciones estocásticas como en Johnson (2000), Guillen (2013) o en Rodríguez, Trejo y Mendoza (2016), que consideran a la convergencia como un proceso estocástico. No obstante, cabría recalcar que, por lo general, estos análisis tienen el limitante de que son puramente empíricos o no son emanados de la teoría propia del modelo de Solow, situación que no es el caso del análisis de las regresiones [1] y [2], considerando a [1] como condición necesaria para [2], como previamente se había explicado.

\section{ESTUDIO DE CONVERGENCIA ESTATAL EN MÉXICO}

Para la estimación correspondiente al presente trabajo se ha utilizado un marco temporal de trece años que componen el periodo de 2003 a 2016, principalmente para observar grosso modo si hay un cambio importante en la distribución del ingreso a partir de la crisis económica de 2008, excluyendo del análisis los años 2017 y 2018; el primero por el reacomodo financiero y productivo que se ocasionó en México por el inicio de la administración de Donald Trump, y el segundo por la efervescencia política provocada por las elecciones más grandes (en magnitud) en la historia de México.

El ingreso per cápita de cada entidad se obtuvo a partir de dividir el PIB de cada entidad (a pesos constantes de $2013^{4}$ entre la población estatal estimada a una tasa constante de crecimiento anual, la cual se calculó mediante la información disponible del Censo de Población y Vivienda del Instituto Nacional de Estadística y Geografía (INEGI), en el que por disponibilidad de datos necesariamente se tuvo que hacer el supuesto de un crecimiento poblacional constante durante todo el periodo de análisis. Cabe hacer notar que el siguiente análisis se encuentra en términos per cápita (al menos que se indique lo contrario).

4 Que se encuentra disponible en el Banco de In-
formación Económica del INEGI. 


\subsection{Ingreso per cápita de las entidades federativas y su dispersión}

A pesar de que se tiene constancia de una tendencia creciente del promedio nacional del ingreso en los primeros años del estudio (2003-2007), éste no superó los \$160200 anuales en todo el periodo, registrándose el nivel más alto del mismo en 2006 y posteriormente tener una caída importante, entrando en su descenso más pronunciado a partir de la crisis de 2008 , para después iniciar una recuperación anual paulatina pero sin llegar a su nivel anterior, ya que de 2012 a 2016 el ingreso promedio nacional se sitúo alrededor de los \$150000 anuales (véase la gráfica 1).

Sólo Baja California, Campeche, Coahuila, Ciudad de México, Nuevo León, Sonora y Tabasco fueron las entidades que superaron consecutivamente cada año el promedio nacional del ingreso.

Cabría hacer especial mención al caso de Campeche, que alcanzó el primer lugar (aunque de forma decreciente) con un promedio del ingreso de $\$ 1032208^{5}$ anuales para todo

5 Este dato es consistente con lo encontrado por Esquivel (1999), ya que en el análisis que presenta hace notar que los datos correspondientes a Campeche y Tabasco se han de tratar como "observaciones irregulares", pues parecen tener un crecimiento del ingreso per cápita "excesivo", y enfatiza en que "este comportamiento se explica en gran medida por la manera en que se ha contabilizado la producción petrolera (...) a esto debe agregarse el efecto económico de los vaivenes de los precios del petróleo que sin duda también han afectado la producción y el ingreso de los estados petroleros" (p. 737).
GRÁFICA 1

NACIONAL DEL INGRESO PER CÁPITA, 2003-2016

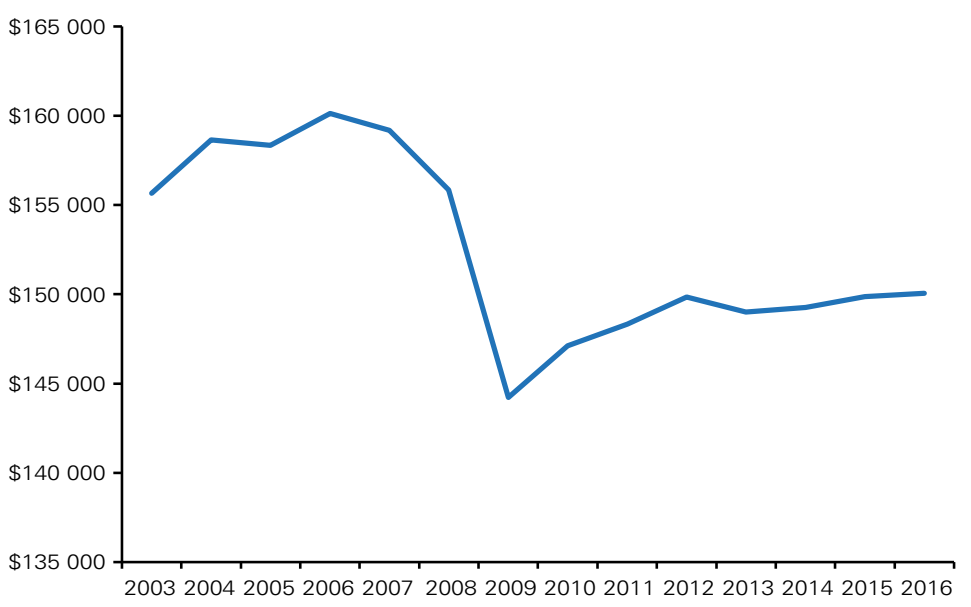

el periodo, seguido por la Ciudad de México con un promedio de \$282 797 y de Nuevo León con \$221 895. Por su parte, Querétaro superó el promedio nacional anual de 2007 a 2016, mientras que Quintana Roo lo hizo durante el mismo periodo pero no de forma consecutiva, es decir, exceptuando 2012, ya que el promedio nacional de ese año fue de \$149 837, mientras que Quintana Roo alcanzó los \$149 755 pesos.

Los tres estados con el menor nivel de ingreso promedio fueron Chiapas, Guerrero y Oaxaca. En el caso de Chiapas, aunque no siempre tuvo un nivel más bajo de forma consecutiva, en comparación con los otros dos estados, fue el de menor promedio anual para todo el periodo con $\$ 56044$, seguido por Oaxaca con un promedio de $\$ 60928$ y Guerrero con \$62 263.

Respecto a la dispersión del ingreso estatal, calculada para cada año mediante la desviación estándar del logaritmo del ingreso, se pueden identificar dos etapas:

- la primera abarcó de 2003 a 2006, se caracterizó porque la dispersión del logaritmo 
del ingreso se mantuvo prácticamente constante, $y$

- la segunda a partir de 2006, donde se observa que anualmente se fue reduciendo dicha dispersión.

A partir de 2008 ha habido un decrecimiento de forma continua y acelerada en comparación a los primeros años, lo que indica que hay evidencia de convergencia $\sigma$, pero para formalizar lo anterior se tendrá que estimar la regresión correspondiente y verificar que el componente de la tendencia determinística sea estadísticamente significativo (véase la gráfica 2).

\subsection{Tasa de crecimiento per cápita de las entidades federativas}

Las cinco entidades que registraron la tasa de crecimiento más elevada para el mismo periodo fueron (de mayor a menor) Zacatecas, Ciudad de México, Aguascalientes, San Luis Potosí y Chihuahua, con una tasa promedio del $12 \%$. Por su parte, Tamaulipas fue el estado con la tasa de crecimiento positiva más baja para el periodo considerado, con un porcentaje muy cercano a cero $(0.07 \%)$.

Sin embargo, existen entidades federativas con tasas de crecimiento negativas: Baja California, Campeche, Tlaxcala y Chiapas. De nuevo cabe hacer especial mención del caso de Campeche, dado que este representa una observación atípica, esto es así porque el promedio de las otras entidades con tasas de crecimiento negativas del ingreso es de $-2.45 \%$; Campeche, por su parte, llega a presentar una tasa de $-33.75 \%$. Es de señalar que, en una primera instancia, el caso de Campeche es consistente con la teoría del crecimiento
GRÁFICA 2

DISPERSIÓN DEL INGRESO PER CÁPITA ESTATAL

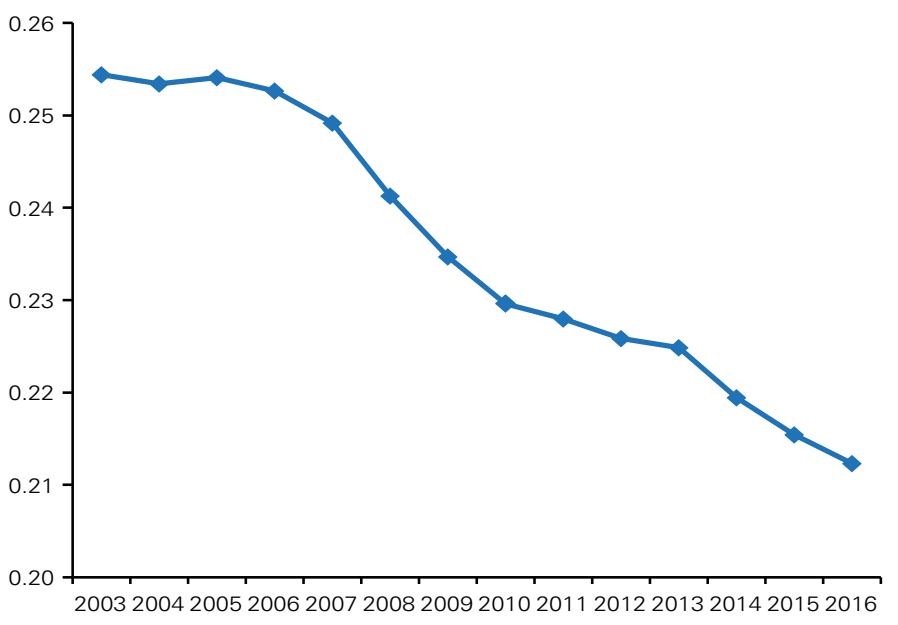

económico que expone el modelo de Solow, ya que al ser una entidad federativa con un nivel inicial muy elevado de ingreso puede esperarse una tasa de crecimiento no sólo baja, sino incluso (como es el caso de esta entidad) negativa, esto sucederá mientras dicha economía se desplaza hacia su estado estacionario. En la gráfica 3 puede apreciarse lo elevado que se encuentra su nivel inicial respecto de las demás entidades federativas.

\section{EVIDENCIA EMPÍRICA}

\subsection{Convergencia $\beta$ absoluta}

La regresión [1] con los datos de las entidades federativas presentó problemas de heterocedasticidad y autocorrelación, por lo que fue necesario utilizar mínimos cuadrados ordinarios (MCO) con un coeficiente consistente con heterocedasticidad y autocorrelación de Newey-West. ${ }^{6}$

6 El método de Newey-West consiste en modificar la matriz de varianzas-covarianzas de los coeficientes estimados mediante el método de Mco, utilizando 
GRÁFICA 3

TASA DE CRECIMIENTO DEL INGRESO PER CÁPITA ESTATAL, 2003-2016

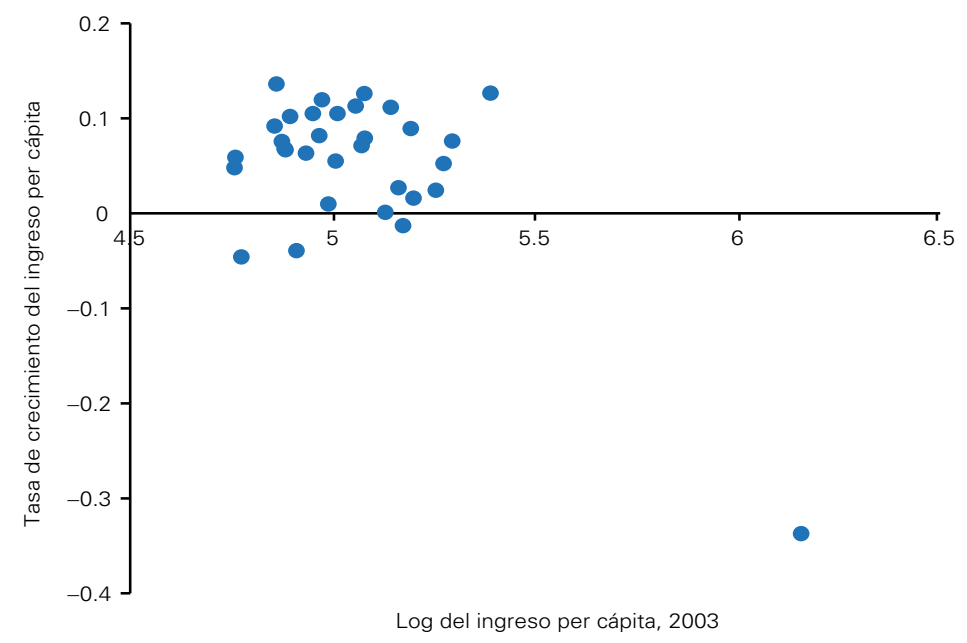

Fuente: elaboración propia.

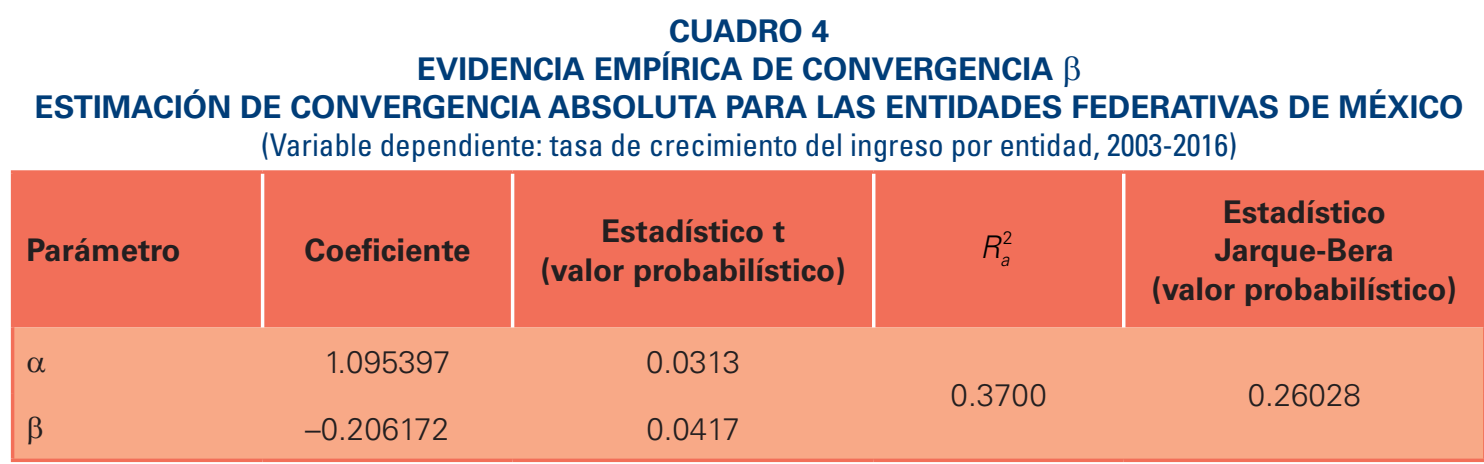

Fuente: elaboración propia con datos del Banco de Información Económica y del Censo de Población y Vivienda de INEGI.

Como puede apreciarse en el cuadro 4, a partir de los resultados obtenidos el valor probabilístico del estadístico Jarque-Bera indica que no se rechaza la hipótesis nula de distribución normal de los residuales, por lo que se puede aplicar la prueba convencional del estadístico $t$ sobre el parámetro $\beta$.

En virtud de que el coeficiente $\beta$ es negativo y es estadísticamente significativo con un intervalo de confianza del 95\%, empíri-

una matriz de varianzas-covarianzas estimada de las perturbaciones estocásticas con factores de ponderación. Por lo anterior, no cambian los valores de los parámetros estimados, pero habrá cambios respecto de su significancia estadística. camente se ha encontrado que existe una relación negativa entre la tasa de crecimiento del ingreso del periodo y su nivel inicial en 2003, por lo que se puede afirmar que hay evidencia estadística sobre convergencia absoluta entre las entidades federativas de México. Es decir, que sin considerar de manera explícita el grado de similitud entre las características estructurales de las entidades, de acuerdo con la teoría el modelo de Solow, hay un solo estado estacionario para todas las economías estudiadas y, por tanto, un solo nivel de ingreso compatible con el estado estacionario, por lo que el ingreso de las mismas convergerá en el largo plazo, 
aunque esto debe confirmarse formalmente mediante el análisis de convergencia $\sigma$.

\subsection{Convergencia $\sigma$}

Ya que se ha encontrado evidencia estadística de convergencia $\beta$ absoluta, entonces se procede a realizar una estimación usando MCO con el fin de comprobar sí existe evidencia de la reducción de la dispersión del ingreso entre las entidades federativas de México. El cuadro 5 muestra los resultados obtenidos de la regresión [2] por MCO utilizando la técnica de Newey-West.

El estadístico Jarque-Bera indicada que no se rechaza la hipótesis nula y, por tanto, que hay evidencia de que los residuales siguen una distribución normal; por su parte, el estadístico $R_{a}^{2}$ indica un buen ajuste de la recta de regresión respecto de los datos.

Con los resultados que se presentan se confirma la significancia estadística del componente del término de tendencia determinística y, además, al ser negativo dicho parámetro muestra que la dispersión del ingreso efectivamente se ha ido reduciendo conforme han venido avanzando los periodos, o bien, expresado en otros términos, se comprueba que existe evidencia de convergencia desde el punto de vista de la dispersión del ingreso.

\section{CONCLUSIONES}

En el aspecto teórico se revisó la importancia de la ecuación de la tasa de crecimiento del capital, ya que sus componentes juegan un papel esencial en la explicación. De forma general, si no existiera un estado estacionario al cual se desplazan las economías, se implicaría que no existe un nivel de ingreso único y, por el contrario, si se establece que hay uno solo para varias, entonces teóricamente se está formalizando el fenómeno de convergencia, ya que en el largo plazo se igualará el nivel de ingreso entre ellas.

La investigación no tenía únicamente un propósito teórico, así que se procedió a explicar el nexo entre el modelo de Solow y el estudio econométrico, planteando una forma sencilla de comprobar el fenómeno sin incluir particularidades como lo requeriría el análisis de convergencia condicional. Asimismo, se prestó especial énfasis a la crítica de Quah (1993) de considerar a la convergencia $\beta$ absoluta como una condición necesaria pero no suficiente.

Para el análisis empírico se utilizaron datos de las entidades federativas de México para el periodo 2003-2016, en los cuales puede observarse que hubo una caída importante en el promedio nacional del ingreso

CUADRO 5

EVIDENCIA EMPÍRICA DE CONVERGENCIA $\sigma$ ESTIMACIÓN DE LATENDENCIA DE DISPERSIÓN PARA LAS ENTIDADES FEDERATIVAS DE MÉXICO

(Variable dependiente: desviación estándar del logaritmo del ingreso de entidad)

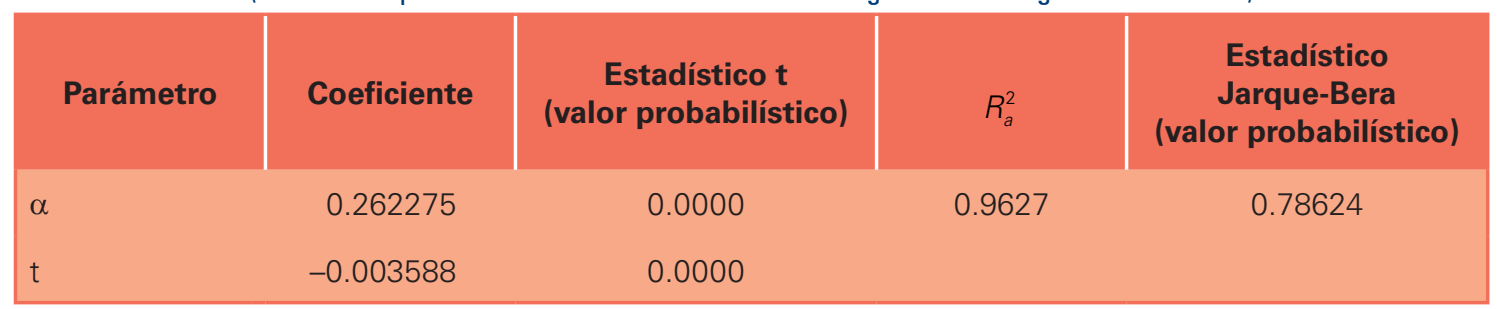

Fuente: elaboración propia con datos de Banco de Información Económica y del Censo de Población y Vivienda de INEGI. 
per cápita a partir de la crisis de 2008. De igual forma, se identificaron dos etapas de la dispersión del ingreso estatal, una que abarca de 2003 a 2006, en la que dispersión del logaritmo del ingreso se mantuvo prácticamente constante, y la segunda a partir de 2006, en la que dicha dispersión se fue reduciendo.

Las entidades federativas que registraron la tasa de crecimiento más elevada fueron $\mathrm{Za}$ catecas, Ciudad de México, Aguascalientes, San Luis Potosí y Chihuahua. Pero también se registraron entidades federativas con tasas de crecimiento negativas: Baja California, Campeche, Tlaxcala y Chiapas. No puede dejar de mencionarse que el estado de Campeche representa una observación atípica, sin embargo, no hay razón teórica económica a priori para que sea excluida como parte de la regresión, en todo caso, en una investigación futura cabría hacer una reflexión más profunda en términos de la métrica petrolera como sostiene Esquivel (1999). Aun así, el caso de Campeche es consistente con la teoría del modelo de Solow, ya que se encontró que dicha entidad poseía un nivel inicial muy elevado de ingreso per cápita y que tenía una tasa de crecimiento negativa para el periodo estudiado.
Posteriormente se procedieron a estimar dos especificaciones, las denominadas regresiones de convergencia $\beta$ absoluta y $\sigma$, una que analiza la relación entre la tasa de crecimiento del ingreso y el ingreso en el momento inicial, y otra que se enfocaba en analizar la dispersión del ingreso. En ambos casos se destacaron los dos estadísticos más importantes para el análisis de convergencia: el estadístico Jarque-Bera y el estadístico $t$, que según los resultados obtenidos en ambas regresiones, las primeras indicaron que se podían realizar las pruebas convencionales t sobre los parámetros y que los estimadores son estadísticamente significativos.

Considerando las limitaciones inherentes del número de datos, la conclusión empírica es que puede afirmarse que existe evidencia de que se cumple la predicción que hace el modelo de Solow en cuanto a la hipótesis de convergencia absoluta, ya que los resultados de las regresiones muestran dos cosas importantes: que existe una relación negativa entre el nivel inicial del ingreso y su tasa de crecimiento, y que la dispersión del mismo se reduce conforme aumentan los periodos analizados. Ambos resultados son consistentes con la existencia de un solo nivel de ingreso per cápita en el estado estacionario. $\mathbf{C}$

\section{REFERENCIAS}

Arellano M. y Bond, S. (1991). Some tests of specification for panel data: Monte Carlo evidence and an application to employment equations. The Review of Economic Studies, 58(2), pp. 277-297. http://dx.doi.org/10.2307/2297968

Arellano, M. y Bover, O. (1995). Another look at the instrumental variable estimation of error-components models. Journal of econometrics, 68(1), pp. 29-51. http:// dx.doi.org/10.1016/0304-4076(94)01642-D

Barro, R.J. y Martin-I-Sala, X., Blanchard, J. y Hall, R.E. (1991). Convergence across states and regions. Broking Papers on Economic Activity, 1991(1), pp. 107-182. http://dx.doi.org/10.2307/2534639

Barro, R., Martin-I-Sala, X. (2004). Economic Growth. Cambridge, MA: The MIT. 
Barro, R., Grilli, V. y Febrero, R. (1997). Macroeconomía: Teoría y política. Madrid: McGraw-Hill.

Blundell, R. y Bond, S. (2000). GMM estimation with persistent panel data: an application to production functions. Econometric Reviews, 19(3), pp. 321-340. http:// dx.doi.org/10.1080/07474930008800475

Camberos, M. y Bracamontes, J. (2016). Is the economic convergence of the Mexican States possible? Estimates for the 1940-2013 period. Journal of Reviews on Global Economics, 5, pp. 48-58.

Cermeño, R. (1998). Performance of various estimators in dynamic panel data models [Documento de trabajo CIDE no. 103]. Centro de Investigación y Docencia Económicas, México.

Cermeño, R. (2001). Decrecimiento y convergencia de los estados mexicanos: un análisis de panel. El Trimestre Económico, 68(272), pp. 603-629.

Claure, B. y Martínez, R. (2016). Sigma convergencia, convergencia beta y condicional en Bolivia, 1990-2011. Una aproximación espacial y de datos de panel. Economía Coyuntural, 1(1), pp. 24-58.

Durlauf S.N. y Johnson, P.A. (2018). Convergence. En: The New Palgrave Dictionary of Economics (pp. 2247-2252). Londres: Palgrave/Macmillan. http://dx.doi. org/10.1057/978-1-349-95189-5_2376

Esquivel, G. (1999). Convergencia regional en México, 1940-1995. El Trimestre Económico, 66(264), pp. 725-762.

Garza, M. (2006). La convergencia regional en España y las causas de convergencia del PIB per cápita en Cataluña. Ensayos, 25(2), pp. 57-80.

German-Soto, V. (2005). Generación del producto interno bruto mexicano por entidad federativa, 1940-1992. El Trimestre Económico, 72(287), pp. 617-653.

Guillen, L. (2013). Crecimiento y convergencia económica: una revisión para Colombia. Revista Dimensión Empresarial, 11(1), pp. 61-76.

Johnson, P.A. (2000). A nonparametric analysis of income convergence across the US States. Economics Letters, 69(2), pp. 219-223. http://dx.doi.org/10.1016/ S0165-1765(00)00299-8

Mankiw, G. (2000). Macroeconomía. Barcelona: Antoni Bosch.

Martin-I-Sala, X. (2000). Apuntes de crecimiento económico. Barcelona: Antoni Bosch.

Quah, D. (1993). Galton's fallacy and tests of the covergence hypotesis. The Scandinavian Journal of Economics, 95(4), pp. 427-443.

Rodríguez D., Trejo, J. y Mendoza, J. (2016). Pair-wise approach to test the regional convergence hypothesis in Mexico. Journal of Reviews on Global Economics, 5, pp. 59-68.

Romer, D. (2002). Macroeconomía avanzada. Madrid: McGraw-Hill.

Solow, R. (1956). A contribution to the theory of economic growth. The Quarterly Journal of Economics, 70(1), pp. 65-84. http://dx.doi.org/10.2307/1884513

Vergara, R., Martínez, A. y Mejía, J. (2010). Crecimiento económico y convergencia regional en el Estado de México. Paradigma Económico, 1, pp. 53-88. 


\section{ANEXO}

INGRESO PER CÁPITA POR ENTIDAD FEDERATIVA, 2003-2016

\begin{tabular}{|c|c|c|c|c|c|c|c|}
\hline Entidad federativa & 2003 & 2004 & 2005 & 2006 & 2007 & 2008 & 2009 \\
\hline Aguascalientes & 119897 & 122385 & 122543 & 127632 & 135782 & 133303 & 123666 \\
\hline Baja California & 149559 & 154632 & 154569 & 158958 & 157116 & 152086 & 132345 \\
\hline Baja California Sur & 158728 & 163416 & 168158 & 173012 & 188360 & 185576 & 177134 \\
\hline Campeche & 1439228 & 1430588 & 1377929 & 1322459 & 1214104 & 1091929 & 966037 \\
\hline Coahuila de Zaragoza & 180044 & 181943 & 182586 & 187798 & 192142 & 187922 & 156068 \\
\hline Colima & 118213 & 116192 & 114885 & 119886 & 125835 & 125848 & 119661 \\
\hline Chiapas & 59569 & 56087 & 55406 & 56139 & 55931 & 56064 & 54607 \\
\hline Chihuahua & 114242 & 118087 & 120691 & 128706 & 131860 & 132266 & 119038 \\
\hline Ciudad de México & 245778 & 255890 & 258739 & 271338 & 274430 & 278411 & 267671 \\
\hline Durango & 101837 & 103743 & 100779 & 103040 & 103287 & 103948 & 101075 \\
\hline Guanajuato & 89531 & 90618 & 89883 & 92911 & 93534 & 94717 & 89234 \\
\hline Guerrero & 57651 & 60179 & 60430 & 61179 & 62219 & 62650 & 59955 \\
\hline Hidalgo & 76192 & 79867 & 77871 & 78661 & 79763 & 81151 & 74689 \\
\hline Jalisco & 120184 & 122002 & 123534 & 128027 & 129973 & 128790 & 120198 \\
\hline México & 76590 & 77300 & 77981 & 80428 & 81590 & 81312 & 76149 \\
\hline Michoacán de Ocampo & 71963 & 72922 & 73487 & 76279 & 77458 & 78276 & 73499 \\
\hline Morelos & 97638 & 97368 & 103021 & 99815 & 99165 & 98052 & 95997 \\
\hline Nayarit & 78718 & 88394 & 90343 & 90733 & 88776 & 93629 & 87176 \\
\hline Nuevo León & 197831 & 205129 & 209622 & 219858 & 228805 & 227930 & 208739 \\
\hline Oaxaca & 57271 & 58876 & 59095 & 59695 & 59584 & 60819 & 59647 \\
\hline Puebla & 75009 & 75913 & 79046 & 81493 & 83792 & 83272 & 75820 \\
\hline Querétaro & 139554 & 146055 & 151863 & 157113 & 160825 & 160519 & 151828 \\
\hline Quintana Roo & 145529 & 151909 & 151986 & 155317 & 164840 & 166423 & 146010 \\
\hline San Luis Potosí & 94168 & 98541 & 101401 & 104843 & 105891 & 106916 & 100121 \\
\hline Sinaloa & 103013 & 108367 & 107823 & 110346 & 113346 & 116318 & 110457 \\
\hline Sonora & 156067 & 161179 & 166650 & 173626 & 172766 & 170145 & 156981 \\
\hline Tabasco & 188406 & 193342 & 204614 & 212776 & 213345 & 219544 & 225302 \\
\hline Tamaulipas & 135089 & 139077 & 145505 & 147328 & 149460 & 153042 & 136865 \\
\hline Tlaxcala & 81570 & 86274 & 74704 & 71978 & 71611 & 73985 & 71243 \\
\hline $\begin{array}{l}\text { Veracruz de Ignacio } \\
\text { de la Llave }\end{array}$ & 86160 & 89502 & 89297 & 93257 & 95158 & 94029 & 91058 \\
\hline Yucatán & 92770 & 95418 & 98240 & 101306 & 102741 & 102086 & 98444 \\
\hline Zacatecas & 72779 & 75107 & 74384 & 78165 & 80057 & 86435 & 88401 \\
\hline
\end{tabular}


INGRESO PER CÁPITA POR ENTIDAD FEDERATIVA, 2003-2016 (continuación)

\begin{tabular}{|c|c|c|c|c|c|c|c|}
\hline Entidad federativa & 2010 & 2011 & 2012 & 2013 & 2014 & 2015 & 2016 \\
\hline Aguascalientes & 128444 & 131111 & 135241 & 136237 & 147217 & 149288 & 160210 \\
\hline Baja California & 135706 & 136398 & 137824 & 137389 & 136420 & 142381 & 145024 \\
\hline Baja California Sur & 173708 & 172886 & 169809 & 159816 & 153234 & 166623 & 164613 \\
\hline Campeche & 916745 & 868062 & 839281 & 832023 & 779279 & 711719 & 661529 \\
\hline Coahuila de Zaragoza & 178269 & 186992 & 192924 & 185591 & 191654 & 190925 & 190386 \\
\hline Colima & 126034 & 132754 & 134215 & 133087 & 133961 & 133869 & 139181 \\
\hline Chiapas & 56496 & 57097 & 57016 & 55131 & 56768 & 54750 & 53556 \\
\hline Chihuahua & 122648 & 124109 & 131871 & 135298 & 136792 & 143175 & 148176 \\
\hline Ciudad de México & 276453 & 285466 & 295912 & 299463 & 304965 & 315992 & 328656 \\
\hline Durango & 103659 & 106689 & 109382 & 111690 & 112980 & 112471 & 115561 \\
\hline Guanajuato & 94264 & 98302 & 100732 & 103214 & 106063 & 111110 & 113966 \\
\hline Guerrero & 62527 & 62688 & 63146 & 62743 & 65046 & 65271 & 65996 \\
\hline Hidalgo & 77412 & 79111 & 80714 & 82222 & 83972 & 87149 & 89114 \\
\hline Jalisco & 125889 & 127728 & 131379 & 132442 & 136032 & 139750 & 144119 \\
\hline México & 80840 & 83335 & 85734 & 86066 & 87314 & 88058 & 89325 \\
\hline Michoacán de Ocampo & 75790 & 78206 & 79500 & 80471 & 85034 & 86155 & 88867 \\
\hline Morelos & 98459 & 96985 & 96269 & 98458 & 98233 & 98154 & 99858 \\
\hline Nayarit & 90127 & 91300 & 89894 & 90905 & 94287 & 97513 & 99492 \\
\hline Nuevo León & 220306 & 225487 & 230259 & 228110 & 231106 & 237835 & 235518 \\
\hline Oaxaca & 59992 & 61181 & 61788 & 62660 & 63307 & 65164 & 63915 \\
\hline Puebla & 81312 & 84258 & 88376 & 86411 & 86127 & 87472 & 89218 \\
\hline Querétaro & 157228 & 164573 & 165184 & 161743 & 170169 & 177336 & 180288 \\
\hline Quintana Roo & 147218 & 149119 & 149755 & 150030 & 149285 & 150474 & 154844 \\
\hline San Luis Potosí & 104195 & 108517 & 112318 & 114967 & 116394 & 120423 & 123941 \\
\hline Sinaloa & 112963 & 114171 & 117238 & 117596 & 119059 & 125184 & 131112 \\
\hline Sonora & 162068 & 173881 & 179566 & 181424 & 181192 & 184216 & 191558 \\
\hline Tabasco & 234527 & 241479 & 243605 & 235132 & 235049 & 229583 & 212411 \\
\hline Tamaulipas & 137129 & 137369 & 137871 & 137522 & 136699 & 137761 & 135305 \\
\hline Tlaxcala & 75910 & 72115 & 73918 & 70667 & 71547 & 74905 & 74514 \\
\hline $\begin{array}{l}\text { Veracruz de Ignacio } \\
\text { de la Llave }\end{array}$ & 93959 & 96728 & 99976 & 99178 & 99375 & 100010 & 99655 \\
\hline Yucatán & 100303 & 102054 & 106226 & 105018 & 106796 & 109348 & 111991 \\
\hline Zacatecas & 97091 & 96256 & 97867 & 95709 & 101380 & 101787 & 99548 \\
\hline
\end{tabular}

Fuente: elaboración propia con datos del Banco de Información Económica sobre el PIB estatal a pesos constantes de 2013 y del Censo de Población y Vivienda 2005 y 2010 del INEGI (en todos los casos se supuso una tasa de crecimiento de población anual constante con base en los datos de los años anteriormente referidos). 
